\title{
Mobility Resolution and Mass Analysis of Ions from Ammonia and Hydrazine Complexes with Ketones Formed in Air at Ambient Pressure
}

\author{
H. R. Bollan \\ Technical Enabling Services Sea Systems Group, Defense Procurement Agency, Bristol, United Kingdom
}

\author{
J. A. Stone \\ Department of Chemistry, Queens University, Kingston, Ontario, Canada
}

\section{J. L. Brokenshire}

Smiths Detection, Waterford, United Kingdom

\author{
J. E. Rodriguez and G. A. Eiceman \\ Department of Chemistry and Biochemistry, New Mexico State University, La Cruces, New Mexico, USA
}

Protonated ammonia and hydrazines $\left(\mathrm{MH}^{+}\right)$form complexes with ketones and the differences in masses and mobilities of the resulting ions, $\mathrm{MH}^{+}$(ketone) ${ }_{n}$, are sufficient for separation in an ion mobility spectrometer at ambient pressure. The highest mass ion for any of the protonated molecules is obtained when the ketone is present at elevated concentrations in the supporting atmosphere of both the source and drift regions of the spectrometer so that an ion maintains a discrete composition and mobility. The sizes of the ion-molecule complexes were found to depend on the number of $\mathrm{H}$ atoms on the protonated nitrogen atom-four for ammonia, three for hydrazine, two for monomethylhydrazine, and one for 1,1-dimethylhydrazine, and the drift times of these ions were proportional to the size of the ion-molecule complex. Unexpected side products, including protonated hydrazones and azines, and associated ketone clusters, were isolated to a single drift tube containing ceramic parts and could not, from CID studies, be attributed to gas-phase ion chemistry. These findings illustrate that mobility resolution of ions in IMS and IMS/MS experiments can be enhanced through chemical modification of the supporting gas atmosphere without changes in the core ion. (J Am Soc Mass Spectrom 2007, 18, 940-951) (C) 2007 American Society for Mass Spectrometry

$\mathrm{H}$ ydrazine and alkyl hydrazines are substances of importance in several specialized propulsion systems, including the nozzle thrusters on the U.S. Space Shuttles and the motors of torpedoes. These highly reactive chemicals are inhalation and dermal irritants, and potential carcinogens with exposure limits in airborne vapors as low as $10 \mathrm{ppb}$. Such standards for occupational hygiene monitoring necessitate measurement methods with low detection limits and high selectivity. Traditional methods of colorimetry and amperometry $[1,2]$ are limited in convenience of use and in specificity by chemical or physical interferences from ammonia, moisture, and other vapors. A selective, sensitive, and fast method for the quantitative determi-

Published online March 28, 2007

Address reprint requests to Dr. G. A. Eiceman, Department of Chemistry and Biochemistry, New Mexico State University, 1175 North Horseshoe Drive, Las Cruces, NM 88003, USA. E-mail: geiceman@nmsu.edu nation of hydrazines has been demonstrated [3, 4] through the gas-phase ionization technique of ion mobility spectrometry (IMS). Such favorable performance can be ascribed to the high proton affinities of hydrazines as found also in atmospheric pressure chemical ionization mass spectrometry (APCI-MS).

Protonated ammonia, hydrazine, and methylhydrazine at trace levels were resolved using IMS through differences in mobility with a drift tube operated at 484 $\mathrm{K}$ [3]. Extension of this concept to a hand-held mobility spectrometer, with a drift tube at ambient temperature, lead to unacceptable resolution of the protonated molecules through formation of hydrated cluster ions without distinctive drift times [4]. Analytically viable separation of ions was sought with acetone as a reagent gas to generate reactant ions $\left(\mathrm{C}_{3} \mathrm{H}_{6} \mathrm{O}\right)_{n} \mathrm{H}^{+}$that would then form ion-molecule complexes containing ammonia or a hydrazine, proton-bound with one or more acetone molecules [4]. Though the resulting ion-molecule com- 
plexes had drift times larger than those for hydrated protonated molecules, the improved stability in drift behavior and peak separations in spectra were insufficient for specific and quantitative determinations. When 5-nonanone was used as a reagent gas, instead of acetone, the reactant ions generated were the protonated molecule $\mathrm{C}_{9} \mathrm{H}_{18} \mathrm{OH}^{+}$and the proton-bound dimer $\left(\mathrm{C}_{9} \mathrm{H}_{18} \mathrm{O}\right)_{2} \mathrm{H}^{+}$. Significantly, the ion-molecule complexes formed by these ions with ammonia and the hydrazines had even longer drift times than those obtained with acetone, and the resolution between peaks in mobility spectra was sufficient for monitoring vapor levels on board the Space Shuttle [4]. Ion drift times and resolution in spectra were shown to be dependent upon the concentration of ketone in the drift gas changing rapidly until $100 \mathrm{ppm}_{\mathrm{v}}$ of ketone in the entire supporting atmosphere of the drift tube. After this and until $1500 \mathrm{ppb}_{\mathrm{v}}$ of ketone, drift times increased slowly and peak shape in spectra sharpened. Mass spectrometry was used to identify the ions in the major product peaks of the IMS spectrum as $\mathrm{MH}^{+}$(ketone) and $\mathrm{MH}^{+}$(ketone $)_{2}(\mathrm{M}=$ ammonia, hydrazine, or methyl hydrazine). These two ions appeared in the single mobility spectrum peak since the complexation reaction of eq 1 remained at equilibrium due to the presence of ketone throughout the mobility spectrometer.

$$
\mathrm{MH}^{+}(\text {ketone })+\text { ketone } \rightleftharpoons \mathrm{MH}^{+}(\text {ketone })_{2}
$$

The reason for the success with a reagent gas was not understood in reference [4] where the observed order of the drift times of the complexes, ammonia $>$ hydrazine $>$ monomethylhydrazine was inverted from the size or mass of the neutral molecules, $\mathrm{M}$.

In this study, the order of drift times for 5-nonanone complexes as shown previously was confirmed as: ammonia $>$ hydrazine $>$ monomethylhydrazine $>$ unsymmetrical dimethylhydrazine. Experiments with a simple drift tube interfaced to a mass spectrometer were used to identify the complexes and other unexpected complexes of protonated hydrazones and protonated azines. Atmospheric pressure chemical ionization (APCI) experiments were used to observe the probable ion-molecule clusters formed in the reactions of protonated hydrazines with 5-nonanone and to investigate possible mechanisms of formation of hydrazone and azine ions, minor constituents in mobility spectra found only in the all ceramic drift tube for IMS/MS studies. Cross-sections of ion-molecule complexes were computed to provide an explanation of the success in resolving the ion-molecule complexes of ammonia and hydrazines with ketones.

\section{Experimental}

The ion mobility spectrometer was a conventional linear design operating at ambient temperature and pressure. Details of construction and operation have been described [5]. TheÅnstrument Åvas Aconstructed Åf Ådis-
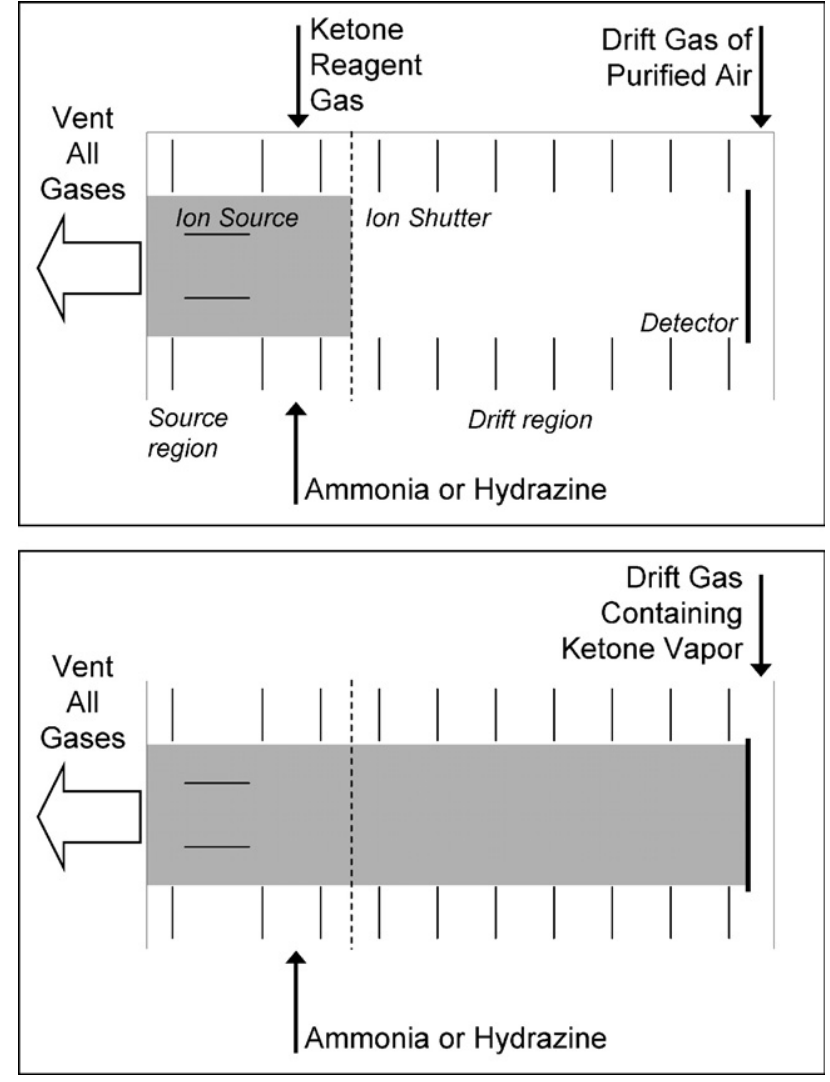

Figure 1. Drift tube of mobility spectrometer with two arrangements for adding ketones as reagent gas and sample vapors. The grey regions show the location of ketone vapors in the drift tube. In the upper frame, ion-molecule complexes formed in the source region are drawn through a purified air atmosphere in the drift region where ion-molecule complexes may dissociate in air alone. In the bottom frame, the ion-molecule complex moves through a chemically uniform composition of gas supporting the equilibrium in eq 1.

crete, alternating Teflon and stainless steel rings with i.d. of $19 \mathrm{~mm}$. Ions, produced by electron emission from a ${ }^{63} \mathrm{Ni}$ source, were gated into a $66 \mathrm{~mm}$ long drift region with a drift field of $244 \mathrm{~V} \mathrm{~cm}^{-1}$ and detected by a Faraday plate and a pico-ammeter. Air was used as both the source and drift gas, and was purified by passing through a $1 \mathrm{~m}$ long tower containing $5 \AA$ molecular sieve; water content in the air was lower than $10 \mathrm{ppm}$.

The flow of air to the source region of the mobility spectrometer was $\sim 50 \mathrm{~mL} \mathrm{~min}{ }^{-1}$ and the drift gas flow from the detector end was $\sim 600 \mathrm{~mL} \mathrm{~min}^{-1}$ as shown in

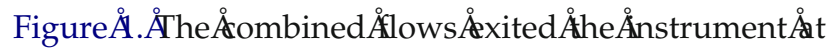
the source end. The requisite concentration of ammonia or one of the hydrazines, introduced to the source, was obtained by dilution of a controlled small flow of air containing the compound into a stream of purified air. Vapor concentrations of each hydrazine were generated using a Teflon permeation tube contained in a chamber held at an appropriate temperature. When required, reagent gas of 5-nonanone was generated in air with permeation tubes, as described above, and introduced 
into the source region or into the drift gas flow as

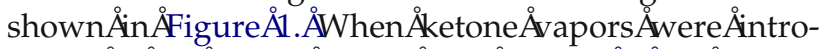

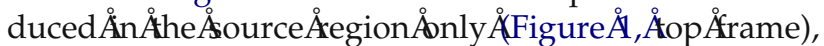
ion complexes formed in the source were drawn by mobility through a purified air atmosphere, where weakly bound complexes might undergo dissociation. In contrast, addition of ketone vapor in at the detector

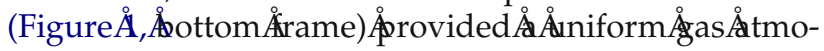
sphere for ion drift, favoring equilibria as shown in eq 1. Concentrations of reagent gas were referenced to a prior study 4$]$ ] Ånd Åudged mobility spectra.

A second, simple mobility spectrometer was constructed, for mass analysis of ion-molecule complexes, from a ceramic tube $1.9 \mathrm{~cm}$ i.d. $\times 9.0 \mathrm{~cm}$ long with equally spaced $1 \mathrm{~cm}$ wide metal bands on the exterior as drift electrodes. The ion source, fields, and flows were analogous to that described above, although the Faraday plate was replaced with a triple quadrupole mass spectrometer (TAGA 6000; Sciex Inc., Toronto, Canada) so that ions could enter the mass spectrometer through a $100 \mu \mathrm{m}$ diameter pinhole, after passage through a nitrogen plenum gas that is characteristic of this interface design. Ammonia or a hydrazine vapors were introduced to the source region in a flow of purified air, and ketone was introduced either in the same flow or

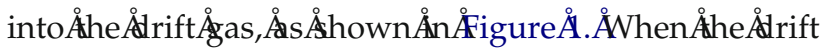
region contained only purified air (and no ketone vapor), some of the weakly bound ion-molecule complexes, formed in the source region, might decompose before reaching the end of the drift region and the inlet of the mass spectrometer.

Since signal intensity was very low in the mass spectrometer, due to the low duty cycle when the mobility spectrometer was in the pulsed mode, experiments were made with the shutter open at all times. All ions therefore entered the mass spectrometer with no time resolution in the mobility scale. A further limitation of the IMS/MS experiments was the upper limit of $450 \mathrm{Da}$ in the mass range of the TAGA 6000 mass spectrometer. Ion-molecule complexes containing more than three 5-nonanone molecules were unobservable due to this limit in mass range. Therefore, the gas-phase interactions of protonated ammonia and the hydrazines were investigated for other symmetrical ketones of lower molar mass including acetone, 3-pentanone, and 4-heptanone to gain an appreciation of the possible sizes of the complexes attainable with 5-nonanone.

Studies were also made with APCI-MS using an API III triple quadrupole instrument (PE-Sciex Inc., Toronto, Canada) equipped with a corona discharge ion source. Vapors of a chemical were passed into the ionization region entrained in ambient air that flowed through a $75 \mathrm{~cm}$ long glass tube, the end of which was placed close to the corona needle. The flow rate of the air was controlled by throttling a rotary pump that draws sample into the source chamber. Two removable glass reservoirs were positioned 20 and $40 \mathrm{~cm}$ from the outer end of the tube in an all-glass manifold. Permeation tubes, one for the substance (hydrazine or ammonia) and one for a ketone, were placed in these glass reservoirs and held at temperatures appropriate for suitable vapor concentrations in the air stream. Collision induced decomposition (CID) spectra were obtained using argon as collision gas to confirm ion-complex identities and to explore if certain ion-complexes could be decomposed to hydrazones or azines.

Reagents or chemicals including 5-nonanone, 4heptanone, 3-pentanone, acetone, hydrazine (HZ), and methylhydrazine $(\mathrm{MMH})$ were obtained from the Aldrich Chemical Co. (Milwaukee, WI) and 1,1Dimethylhydrazine was obtained from Fluka (Milwaukee, WI). All were used without further purification or treatment. Ammonia was produced by evaporation from ammonium hydroxide solution.

\section{Computations}

Computational software (HyperChem 4.5; Waterloo, Ontario, Canada) was used to create models for clusters comprising protonated ammonia or a protonated hydrazine and 5-nonanone. Geometries were optimized using semiempirical PM3 parameters with the PolakRibiere optimizer algorithm.

\section{Results and Discussion}

\section{Ion Mobility Spectrometry Studies with 5-Nonanone}

Experiments were undertaken with the ion mobility spectrometer to confirm the previously-reported limiting mobilities for the protonated ammonia and proton-

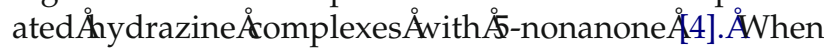
the instrument was operated with dry air alone, there was only one significant peak in the spectrum, the well-studied reactant ion peak (RIP), the hydrated pro-

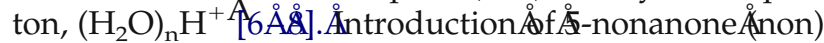
to the source resulted in the appearance of two new peaks and diminution of the hydrated proton peak. The new peaks are attributable to the protonated ketone, (non) $\mathrm{H}^{+}$, and hydrates (at $12.50 \mathrm{~ms}$ ) and the protonbound ketone dimer, (non) ${ }_{2} \mathrm{H}^{+}$(at $16.55 \mathrm{~ms}$ ). This identification is based on previous IMS/MS studies with $\AA$ honanone 4 4]

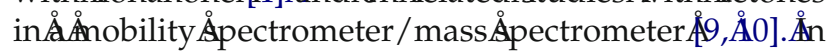
IMS, ions of a substance are characterized by drift velocity $\mathrm{v}_{\mathrm{d}}$ for an ion swarm and by the related mobility coefficient. Mobility coefficients are derived from the drift length, 1 , and drift time for the ion swarm, $t_{d}$ (eq 2):

$$
\mathrm{K}=\frac{\mathrm{v}_{\mathrm{d}}}{\mathrm{E}}=\frac{1}{\mathrm{t}_{\mathrm{d}} \mathrm{E}}
$$

where $\mathrm{E}$ is the electric field of the drift region. Values for $\mathrm{K}$ are normalized to standard temperature and pressure (eq 3) yielding the reduced mobility coefficient $\left(\mathrm{K}_{\mathrm{o}}\right)$ in eq 3 : 


$$
\mathrm{K}_{\mathrm{o}}=\mathrm{K}\left(\frac{\mathrm{p}}{760}\right)\left(\frac{273}{\mathrm{~T}}\right)
$$

where $\mathrm{p}$ is the pressure in $\mathrm{mm} \mathrm{Hg}$ and $\mathrm{T}$ is absolute temperature of the drift gas or supporting atmosphere in the drift tube.

The values for $\mathrm{K}_{\mathrm{o}}$, computed here as $1.61 \mathrm{~cm}^{2} \mathrm{~V}^{-1} \mathrm{~s}^{-1}$ for (non) $\mathrm{H}^{+}$and $1.21 \mathrm{~cm}^{2} \mathrm{~V}^{-1} \mathrm{~s}^{-1}$ for (non) ${ }_{2} \mathrm{H}^{+}$, are in excellent agreement with previous values of 1.61 $\mathrm{cm}^{2} \mathrm{~V}^{-1} \mathrm{~s}^{-1}$ and $1.15 \mathrm{~cm}^{2} \mathrm{~V}^{-1} \mathrm{~s}^{-1}$ obtained in this laboratory with another mobility spectrometer operat-

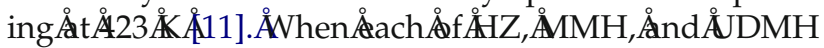
was introduced separately into the source region without ketone reagent gas, the RIP decreased and a new peak appeared. This peak was situated close to the reactant ion peak and would be $(\mathrm{HZ}) \mathrm{H}^{+}$for hydrazine, $(\mathrm{MMH}) \mathrm{H}^{+}$for monomethylhydrazine, and (UDMH) $\mathrm{H}^{+}$ for 1,1-dimethylhydrazine. The proton affinities of all these substances are higher than those of water, and proton transfer from the reactant ion $\left(\mathrm{H}_{2} \mathrm{O}\right)_{n} \mathrm{H}^{+}$to the substances was facile. Although the ions had comparable mobility coefficients, affording no resolution between peaks and little distinction for identification of a vapor, the ions could be adducted with ketone added as a reagent gas to the supporting atmosphere inside the drift tube and separated by differences in mobility of the ion-molecule complexes. Detailed studies were made by IMS first with the ammonia with 5-nonanone as a model system. Studies were then extended to include the hydrazines with ketones. The findings with ammonia were generally descriptive of the behavior of all hydrazines.

\section{Ion Mobility Spectrometry Studies of Ammonia with 5-Nonanone}

Sufficient ammonia was introduced to the ion source so that the RIP disappeared and a single peak was observed at a drift time of $7.90 \mathrm{~ms}$; this was attributed to $\left(\mathrm{NH}_{3}\right)_{n} \mathrm{H}^{+}$and its hydrates (peak $\mathrm{A}$, spectrum a, Figure ̊2). Åmmonia Ån Ålow ÅconcentrationÅwillÅreact with the $\left(\mathrm{H}_{2} \mathrm{O}\right)_{n} \mathrm{H}^{+}$reactant ion in the ion source by proton transfer and an accompanying transfer of water molecules (eq 4):

$$
\left(\mathrm{H}_{2} \mathrm{O}\right)_{\mathrm{n}} \mathrm{H}^{+}+\mathrm{NH}_{3} \rightarrow\left(\mathrm{H}_{2} \mathrm{O}\right)_{(\mathrm{n}-\mathrm{x})} \mathrm{NH}_{4}^{+}+\mathrm{xH}_{2} \mathrm{O}
$$

An increase in ammonia concentration will result in more displacement of water by ammonia (eq 5):

$$
\begin{aligned}
& \left(\mathrm{H}_{2} \mathrm{O}\right)_{(\mathrm{n}-\mathrm{x})} \mathrm{NH}_{4}^{+}+\mathrm{mNH}_{3} \\
& \quad \rightarrow\left(\mathrm{H}_{2} \mathrm{O}\right)_{(\mathrm{n}-\mathrm{x}-\mathrm{y})}\left(\mathrm{NH}_{3}\right)_{(\mathrm{m}+1)} \mathrm{H}^{+}+\mathrm{yH}_{2} \mathrm{O}
\end{aligned}
$$

although levels of ammonia were kept low in experiments here so that cluster ions with multiple ammonia molecules were not formed per eq 5. The extent of hydration of the protonated ammonia cluster in eq 4 was unknown, but the use of dried air for the supporting

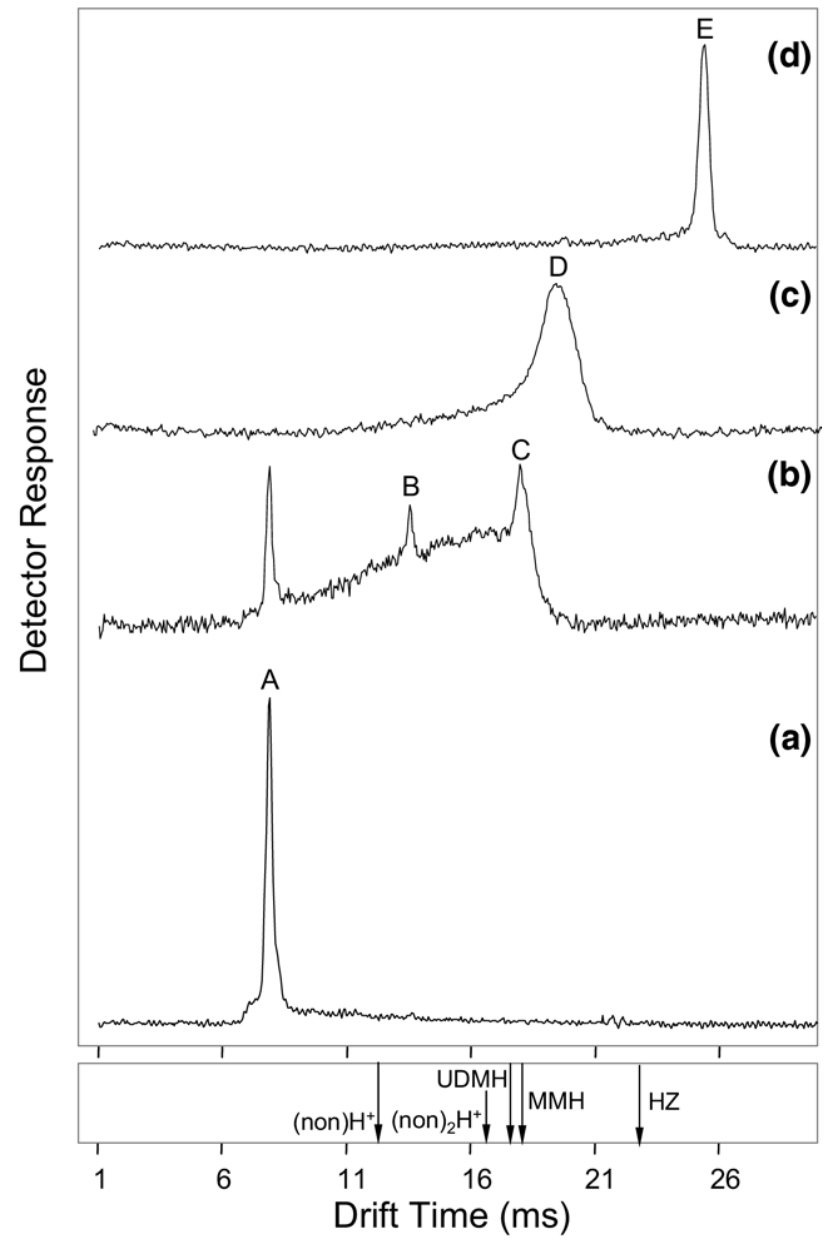

Figure 2. Ion mobility spectra obtained for the $\mathrm{NH}_{3}$ : 5-nonanone system when the ketone was present throughout the source and drift regions. The peaks are identified as: $\mathrm{A}, \mathrm{NH}_{4}^{+} ; \mathrm{B}$, $\mathrm{NH}_{4}^{+}$(non); $\mathrm{C}, \mathrm{NH}_{4}^{+}$(non) $)_{2}$, D, see text; and $\mathrm{E}, \mathrm{NH}_{4}^{+}$(non) $)_{4}$ in the top frame. In the bottom frame, the drift times for other hydrazines are shown under conditions used for peak E.

atmosphere in the entire drift tube should ensure that it is small. In further discussion, the $\left(\mathrm{H}_{2} \mathrm{O}\right)_{(\mathrm{n}-\mathrm{x}-\mathrm{y})}\left(\mathrm{NH}_{3}\right)_{\mathrm{m}} \mathrm{H}^{+}$ cluster is written as $\mathrm{NH}_{4}^{+}$, and the possible hydration and solvation of other ions are assumed but not acknowledged in the formula. In these studies, $\mathrm{NH}_{4}^{+}$was considered the reactant ion for experiments and 5-nonanone was added as a reagent gas.

The introduction of increasing, but not determined, amounts of 5-nonanone (non) at the detector end of the instrument and, thus, through both drift and source regions, led to a decrease in intensity of the reactant ion peak (peak A) and the appearance of two new peaks labeled B and C with drift times of $13.50 \mathrm{~ms}$ and 17.75角s Åspectrum baseline in the spectrum between peak $A$ and peak $B$ that continues to peak C. A well defined, narrow peak in a mobility spectrum with constant drift time is due generally to ions formed in the source region, gated into the drift region, and subsequently unchanged in structure during passage to the detector. Ions that change 
structure and hence mobility in the drift region do not generate such discrete peaks and are evident as a raised baseline between peaks. The interpretation of the positive slope of the baseline is that ions of peak A are reacting with 5-nonanone to form ions in peak $B$, which are further reacting with 5-nonanone to form ions in peak $C$. The ions were not positively identified but are, by inference: peak $\mathrm{B}, \mathrm{NH}_{4}^{+}$(non) (at $13.50 \mathrm{~ms}$ ) and peak C. $\mathrm{NH}_{4}^{+}$(non) 2 (at $17.75 \mathrm{~ms}$ ), and are formed in association reactions as shown in eq 6 and eq 7 :

$$
\begin{aligned}
& \underset{\text { peak A }}{\mathrm{NH}_{4}^{+}}+\text {non } \rightarrow \underset{\text { peak B }}{\mathrm{NH}_{4}^{+}(\text {non })} \\
& \underset{\text { peak B }}{\mathrm{NH}_{4}^{+}(\text {non })}+\text { non } \rightarrow \underset{\text { peak C }}{\mathrm{NH}_{4}^{+}(\text {non })_{2}}
\end{aligned}
$$

The mobility of an ion in electric fields in IMS drift tubes depends on the inverse of both its collision cross

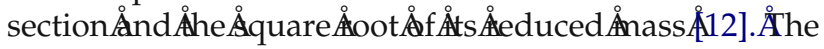
ions $\mathrm{NH}_{4}^{+}$(non) and $\mathrm{H}^{+}$(non) have almost identical reduced masses in air, although the collision cross section of $\mathrm{NH}_{4}^{+}$(non) is expected to be slightly greater than that of $\mathrm{H}^{+}$(non). The mobility of $\mathrm{H}^{+}$(non) should then be slightly greater than that of $\mathrm{NH}_{4}^{+}$(non). This corresponds to a drift time for $\mathrm{NH}_{4}^{+}$(non) longer than for $\mathrm{H}^{+}$(non) and consistent with the observed drift times of 13.50 and $12.50 \mathrm{~ms}$, respectively. The same argument holds for the assignment of the second peak (C) at $17.75 \mathrm{~ms}$ as $\mathrm{NH}_{4}^{+}$(non) $)_{2}$ from comparison with $\mathrm{H}^{+}$(non) $)_{2}$ at $16.55 \mathrm{~ms}$.

Reactions in eq 6 and eq 7 occur both in the source and drift regions when the same concentration of ketone is presence in each. Thus, any ion appearing in the spectrum between peaks $\mathrm{A}$ and $\mathrm{B}$ would have spent the first part of its transit time as $\mathrm{NH}_{4}^{+}$and the final part as $\mathrm{NH}_{4}^{+}$(non). The drift time would be intermediate between those of the two ions. There is a whole spectrum of such ions, the positive slope of the ion intensity between peaks $\mathrm{A}$ and $\mathrm{B}$ formed as a result of the kinetics of the process. The intensity of $\mathrm{NH}_{4}^{+}$is greatest at the ion shutter and decreases, via the reaction in eq 6 , along the length of the drift region. The rate of reaction therefore decreases with drift distance, and ions that spend less time as $\mathrm{NH}_{4}^{+}$and more time as $\mathrm{NH}_{4}^{+}$(non) have a greater intensity than those spending more time as $\mathrm{NH}_{4}^{+}$and will appear in the spectrum closer to the $\mathrm{NH}_{4}^{+}$(non) peak. The result is evident as the rising baseline between peaks A and B. The same interpretation can be applied to the spectral pattern between peaks $B$ and $C$ that is due mainly to the reaction described in eq 7.

As the concentration of 5-nonanone was increased further, the drift times of all three peaks remained constant, but peak A declined in intensity as the other two ( $B$ and $C$ ) increased. With still higher 5-nonanone concentration, peak A disappeared from the spectrum, followed by the disappearance eventually also of peak B. All these changes were gradual in the transformations of spectra suggesting displacements of equilibria.
Up to this point, the drift time and symmetry of peak C, due to $\mathrm{NH}_{4}^{+}(\text {non) })_{2}$, was constant; however, peak $\mathrm{C}$ became broad and asymmetrical, and the drift time increased to the location of peak D (at $20.27 \mathrm{~ms}$ ) with further increases in ketone concentrations (spectrum c

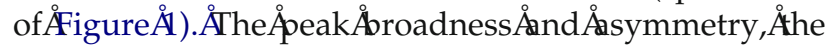
latter more pronounced towards shorter drift times, is symptomatic of transformations in the drift region, here the formation of ion-molecule complexes. Since the drift time associated with the peak maximum is greater than that for peak $C$, the associated ion(s) is slower and must haveÅaÅlargerÅcollision Åcross Åsection $\AA[$, Å8] $\mathrm{NH}_{4}^{+}$(non) ${ }_{2}$; thus, the ammonium-ketone complexes should contain at least three molecules of the ketone during some time the drift period.

A still further increase in the concentration of 5-nonanone led to a sharp, symmetrical peak at 25.45

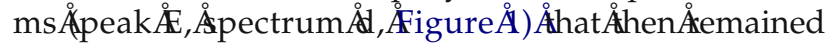
invariant with additional increases in ketone vapor concentrations. As discussed above, this sharp peak denotes the presence of a major single ion, $\mathrm{NH}_{4}^{+}(\text {non })_{n}$, of specific character that was formed in the source and traversed the drift region unchanged. The identities of the ions appearing in D and E may be surmised from the linear correlation of drift time with ion mass for a

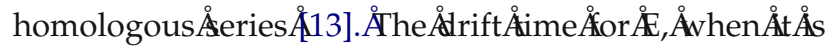
assumed to be $\mathrm{NH}_{4}^{+}$(non) $)_{4}$, gives a linear fit with those for $B$ and $C$ and an equation for the line, $t_{d}(m s)=$ $0.0279 \mathrm{u}+9.148$. The expected drift time for $\mathrm{NH}_{4}^{+}(\text {non })_{3}$ (mass of $445 \mathrm{u}$ ) from this analysis should be $21.56 \mathrm{~ms}$ and so the peak D, with a drift time of $20.27 \mathrm{~ms}$, was comprised mainly of ions that have spent $32 \%$ of drift time as $\mathrm{NH}_{4}^{+}$(non) $)_{3}$ and $68 \%$ as $\mathrm{NH}_{4}^{+}$(non) $)_{4}$.

In their initial work with ammonia and the hydrazines, Eiceman et al. reported that the mobility of the complex for the ammonium ion and 5-nonanone had a drift time of $13.46 \mathrm{~ms}$, and that $\mathrm{NH}_{4}^{+}$(non) and $\mathrm{NH}_{4}^{+}$(non) $)_{2}$ were found in IMS/MS/MS measurements

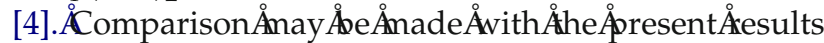
using $\mathrm{K}_{\mathrm{o}}$ values obtained from drift times and eq 2 and eq 3 . The drift length of the spectrometer used in

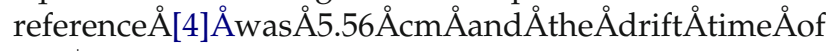
$\mathrm{NH}_{4}^{+}(\text {non })_{\mathrm{n}}$ at $1500 \mathrm{ppb}$ of 5-nonanone was $13.46 \mathrm{~ms}$, giving a derived $\mathrm{K}_{\mathrm{o}}$ of $1.36 \mathrm{~cm}^{2} \mathrm{~V}^{-1} \mathrm{~s}^{-1}$. This value denotes an ion mass of $287 \mathrm{Da}$ from interpolation of ion mass versus $\mathrm{K}_{\mathrm{o}}$ obtained in the present work and is close to that of $\mathrm{NH}_{4}^{+}$(non) $)_{2}, 302 \mathrm{Da}$.

\section{Ion Mobility Spectrometry Studies of Hydrazines with 5-Nonanone}

Experiments were completed for each of HZ, MMH, and UDMH in a supporting atmosphere modified with 5-nonanone and spectra for each exhibited a tendency to a single product ion peak with drift time denoted by

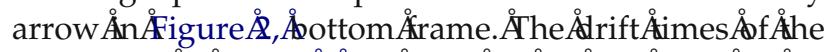

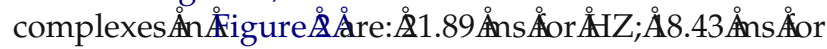
$\mathrm{MMH}$, and $18.3 \mathrm{~ms}$ for UDMH. As the concentration of 
5-nonanone was increased, drift times showed little change over an extended range of concentration. Spectra were more complex than those obtained with ammonia and contained the reactant ion, $(\text { non })_{n} \mathrm{H}^{+}$, as the dominant peak and a broad baseline profile, consistent

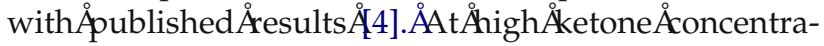
tions when ammonia gave the single, very narrow peak (EÅofÅFigure Å2), ÅtheÅpeaks Åfor ÅtheÅhydrazinesÅwere broadened, poorly defined, and increased in drift times. The order of the drift times at high ketone concentration in the present study, $\mathrm{NH}_{4}^{+}>(\mathrm{HZ}) \mathrm{H}^{+}>(\mathrm{MMH}) \mathrm{H}^{+}>$ $(\mathrm{UDMH}) \mathrm{H}^{+}$, in the same 5-nonanone concentration, was $\begin{aligned} & \text { he } \\ & \text { same }\end{aligned}$ $1.08 \mathrm{~cm}^{2} \mathrm{~V}^{-1} \mathrm{~s}^{-1}$ for $\mathrm{HZ}$ and $1.28 \mathrm{~cm}^{2} \mathrm{~V}^{-1} \mathrm{~s}^{-1}$ for $\mathrm{MMH}$ are lower than those derived from the data in reference [4] shows that the masses for ion-molecule complexes here are higher than in the previous study, meaning that concentrations here exceeded $1.5 \mathrm{ppm}_{\mathrm{v}}$ of ketone in drift gas. Mass identification of ions formed between ammonia and hydrazines in ketone rich atmospheres were sought using IMS/MS/MS experiments.

\section{IMS/MS/MS Studies of Hydrazines with 5-Nonanone}

SpectraÅshownÅin ̊̊FigureÅ3ÅforÅhydrazineÅand $\AA$ 3pentanone were obtained when the ketone was

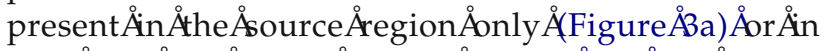

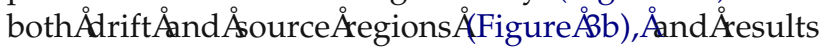
were typical of other combination of ketones with hydrazines. Protonated hydrazine solvated by 3,2 , and 1 ketone molecules at $m / z 291,205$, and 119, respec-

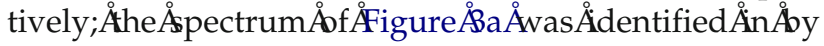
CID studies. The $\mathrm{m} / \mathrm{z} 291$ peak is very low in intensity. The CID spectrum of the peak with highest intensity in

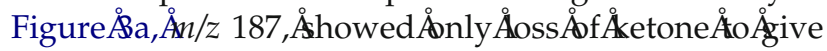
$\mathrm{m} / \mathrm{z} 101$, an ion also present in the figure. The ion for $\mathrm{m} / \mathrm{z}$ 101 was not collisionally dissociated up to $10 \mathrm{eV}$. Similar stable ions were observed when other ketones were introduced into source containing hydrazine: $\mathrm{m} / \mathrm{z}$ 73 with acetone, $m / z 129$ with 4-heptanone, and $m / z 157$ with 5-nonanone. Ions solvated by two or more ketone molecules were also present in the spectra; for example, m/z 273Åin ÅFigureẢ3å̊is $\mathrm{m} / z$ 101Åsolvated Åby Åtwo 3-pentanone molecules. The CID spectrum of the ion of

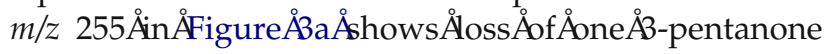
molecule to give the very stable $\mathrm{m} / \mathrm{z} 169$. Analogous stable ions, sometimes solvated by a single ketone molecule, observed in the spectra of the other ketones were: $\mathrm{m} / \mathrm{z} 113$ with acetone; $\mathrm{m} / \mathrm{z} 225$ with 4-heptanone, and $m / z 281$ with 5-nonanone.

The identities of $\mathrm{m} / \mathrm{z} 101$ and 169 observed in spectra obtained with the hydrazine:3-pentanone system and the corresponding ones obtained with the other ketones can be determined by inference. Ions at $\mathrm{m} / \mathrm{z} 73, \mathrm{~m} / \mathrm{z} 101, \mathrm{~m} / \mathrm{z}$ 129 , and $m / z 157$ are all equivalent to [HZ.H.ketone -

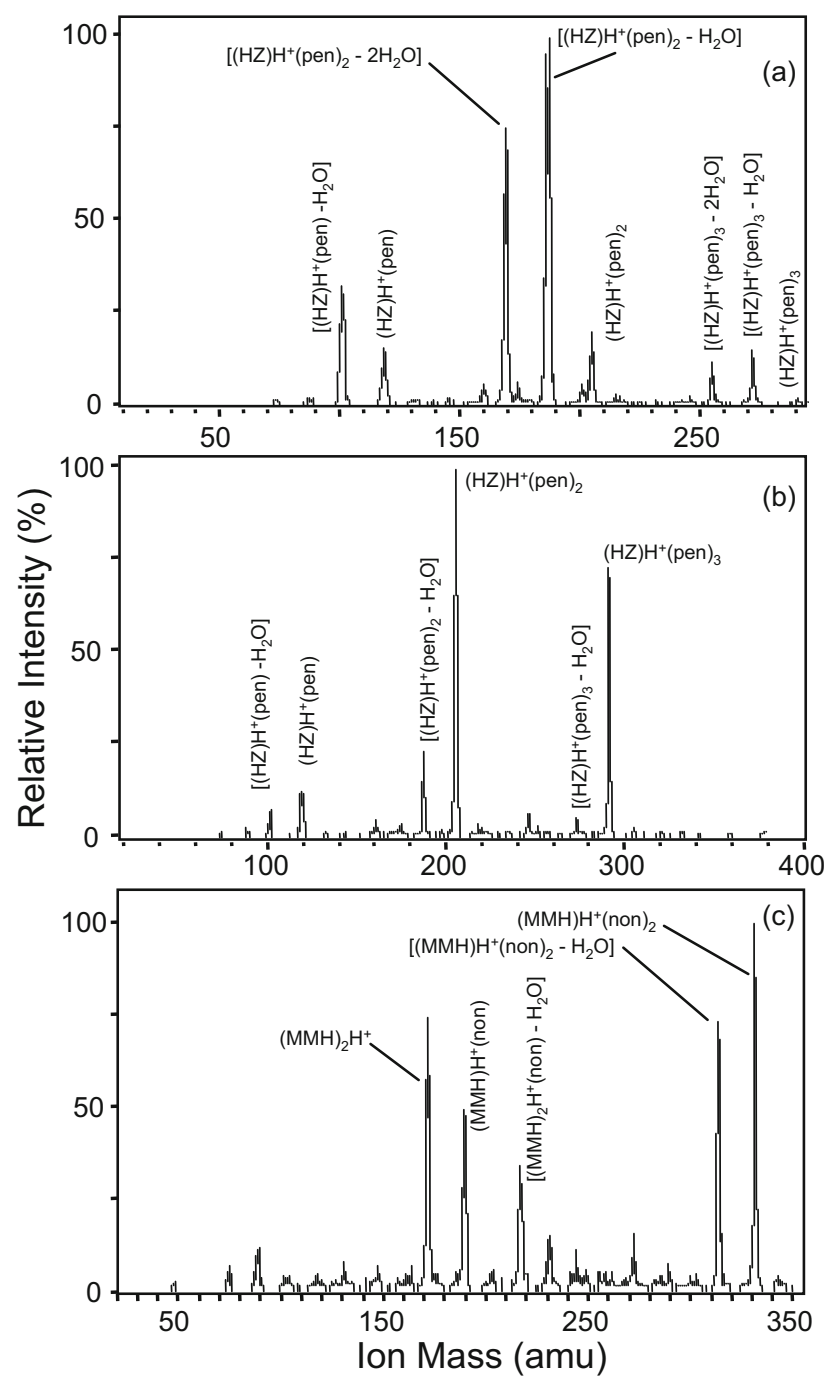

Figure 3. Mass spectra obtained for hydrazine (HZ) with (a) 3-pentanone (pen) only in the source region and (b) pen 3-pentanone throughout the drift tube. Mass spectrum for methyl hydrazine $(\mathrm{MMH})$ with 5-nonanone (non) through the drift tube (c).

$\left.\mathrm{H}_{2} \mathrm{O}\right]^{+}$, i.e., a protonated hydrazone. This is shown in eq 8 for 3-pentanone:

$$
\mathrm{H}_{2} \mathrm{NNH}_{3}^{+}+\mathrm{OC}\left(\mathrm{C}_{2} \mathrm{H}_{5}\right)_{2} \rightarrow \mathrm{H}_{2} \mathrm{NN}\left(\mathrm{H}^{+}\right) \mathrm{C}\left(\mathrm{C}_{2} \mathrm{H}_{5}\right)_{2}+\mathrm{H}_{2} \mathrm{O}
$$

The ions $\mathrm{m} / \mathrm{z} 113, \mathrm{~m} / \mathrm{z} 169, \mathrm{~m} / \mathrm{z} 225$, and $\mathrm{m} / \mathrm{z} 281$ correspond to [HZ: $\mathrm{H}^{+}$.(ketone $\left.)_{2}-2 \mathrm{H}_{2} \mathrm{O}\right]$, i.e., a protonated azine. These ions were formed from the dissociative addition of a further ketone to the protonated hydrazone is shown in eq 9:

$$
\begin{aligned}
& \left(\mathrm{C}_{2} \mathrm{H}_{5}\right)_{2} \mathrm{CO}+\mathrm{H}_{2} \mathrm{NN}\left(\mathrm{H}^{+}\right) \mathrm{C}\left(\mathrm{C}_{2} \mathrm{H}_{5}\right)_{2} \\
& \quad \rightarrow\left(\mathrm{C}_{2} \mathrm{H}_{5}\right)_{2} \mathrm{CNN}\left(\mathrm{H}^{+}\right) \mathrm{C}\left(\mathrm{C}_{2} \mathrm{H}_{5}\right)_{2}+2 \mathrm{H}_{2} \mathrm{O}
\end{aligned}
$$

When ketone was present throughout the whole instrument, hydrazine formation was minor and the proton- 
Table 1. Highest number of ketones observed solvating $\mathrm{MH}^{+}$for homologous series of ketones with 3 to 11 carbons

\begin{tabular}{|c|c|c|c|c|c|}
\hline M & Point of introduction of ketone ${ }^{a}$ & $\left(\mathrm{CH}_{3}\right)_{2} \mathrm{CO}$ & $\left(\mathrm{C}_{2} \mathrm{H}_{5}\right)_{2} \mathrm{CO}$ & $\left(\mathrm{C}_{3} \mathrm{H}_{7}\right)_{2} \mathrm{CO}$ & $\left(\mathrm{C}_{5} \mathrm{H}_{9}\right)_{2} \mathrm{CO}$ \\
\hline \multirow[t]{2}{*}{$\mathrm{NH}_{3}$} & source & 2 & 2 & 2 & 2 \\
\hline & detector & 4 & 4 & $3^{\mathrm{b}}$ & $3^{b}$ \\
\hline \multirow[t]{2}{*}{ Hydrazine } & source & 2 & 3 & 1 & 2 \\
\hline & detector & 4 & 3 & 3 & $3^{c}$ \\
\hline \multirow[t]{2}{*}{$\mathrm{MMH}$} & source & 2 & 2 & 2 & 1 \\
\hline & detector & 3 & $3^{d}$ & $3^{d}$ & 2 \\
\hline \multirow[t]{2}{*}{ UDMH } & source & 1 & 1 & 1 & 1 \\
\hline & detector & 2 & 2 & 1 & 1 \\
\hline \multirow[t]{2}{*}{ Tetramethyl-hydrazine } & source & 1 & 1 & 1 & 1 \\
\hline & detector & 1 & $2^{d}$ & 1 & $2^{d}$ \\
\hline
\end{tabular}

${ }^{a}$ Reference to Figure 1, where top and bottom frames, respectively, refer to source or detector.

${ }^{b}$ Number measured limited by mass range of mass spectrometer and could have been 4 .

'Intensity too minor for positive CID identification.

ated hydrazine/ketone ureÅ3b).ÅTheÅmajor Åpeaks ÅareÅdueÅtoÅprotonated hydrazine solvated by two and three ketone molecules, $\mathrm{HZ} \cdot \mathrm{H}^{+} \cdot\left(\mathrm{OC}\left[\mathrm{C}_{2} \mathrm{H}_{5}\right]_{2}\right)_{2}$ at $m / z 205$ and $\mathrm{HZ} \cdot \mathrm{H}^{+} \cdot\left(\mathrm{OC}\left[\mathrm{C}_{2} \mathrm{H}_{5}\right]_{2}\right)_{3}$ at $m / z$ 291. The hydrazine adduct, $\mathrm{HZ} \cdot \mathrm{H}^{+} \cdot \mathrm{OC}\left(\mathrm{C}_{2} \mathrm{H}_{5}\right)_{2}$, is present at $\mathrm{m} / \mathrm{z} 119$, and there is a trace peak at $\mathrm{m} / \mathrm{z} 377$ due to $\mathrm{HZ} \cdot \mathrm{H}^{+} \cdot\left(\mathrm{OC}\left[\mathrm{C}_{2} \mathrm{H}_{5}\right]_{2}\right)_{4}$. Protonated hydrazone and protonated hydrazine peaks are still present but their relative importance is severely diminished. The most intense ion of these types is the protonated hydrazone, $\mathrm{H}_{2} \mathrm{NN}\left(\mathrm{H}^{+}\right) \mathrm{C}\left(\mathrm{C}_{2} \mathrm{H}_{5}\right)_{2}$, at $m / z$ 187. All the ketones except 5-nonanone had one of the solvated hydrazinium ions as the major peak when the ketone was present throughout the instrument. For 5-nonanone, the largest peak was always $m / z 299,\left(\mathrm{C}_{9} \mathrm{H}_{20} \mathrm{~N}_{2}\right) \mathrm{H}^{+}\left(\mathrm{C}_{9} \mathrm{H}_{18} \mathrm{O}\right)$, the solvated protonated hydrazone. The adduct ion $\mathrm{HZ} \cdot \mathrm{H}^{+} \cdot(\text { non })_{2}$ was the second largest peak, and there was a very small peak at $m / z 459$ that could have been $\mathrm{HZ} \cdot \mathrm{H}^{+}$.(non) $)_{3}$, but the intensity was too low for confirmation. Unfortunately, an ion-molecule complex with four ketones exceeded the range of the mass spectrometer and necessitated further studies by APCI MS with a mass range to $\mathrm{m} / \mathrm{z} 2000$.

When methylhydrazine was substituted for hydrazine, $\mathrm{MMH} \cdot \mathrm{H}^{+}$.(ketone) $)_{2}$ was the most intense ion in the spectra for 3-pentanone and 4-heptanone when present throughout the drift tube. In the latter case, the protonated hydrazone $\left[\mathrm{MMH} \cdot \mathrm{H}^{+} \cdot\right.$ (ketone) $-\mathrm{H}_{2} \mathrm{O}$ ] solvated by one and two ketone molecules was the only other significant ion. Acetone showed a similar behavior but $\mathrm{MMH} \cdot \mathrm{H}^{+}$.(ketone) $)_{3}$ was of also present. Although protonated hydrazones and their ketone adducts were severely diminished in intensity with ketones throughout the instrument, 5-nonanone was

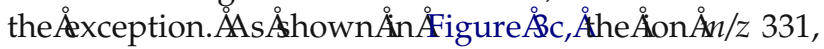
$\mathrm{MMH} \cdot \mathrm{H}^{+} \cdot\left(\mathrm{C}_{9} \mathrm{H}_{18} \mathrm{O}\right)_{2}$, is the most intense peak in the spectrum but the protonated hydrazone and its solvate, $\mathrm{m} / \mathrm{z} 171$ and 313, are also of high intensity. No azine was observed.

The chemical UDMH behaved in a similar manner to MMH in that the spectra contained the protonated molecule solvated by ketone and protonated hydrazones. The latter ions were considerably reduced in relative intensity when ketone was introduced throughout the drift region. The most intense peak was protonated UDMH solvated by one ketone. Tetramethylhydrazine, TMH $\left(\mathrm{CH}_{3}\right)_{2} \mathrm{NN}\left(\mathrm{CH}_{3}\right)_{2}$, produced the most simple spectrum, where only the protonated molecule, the proton-bound dimer, and the protonated molecule solvated by one ketone molecule were observed. All

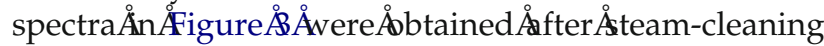
the ceramic drift tube between experiments with different hydrazines. Nonetheless, ions at low intensity were sometimes observed that could be attributed to previous samples.

\section{Interpretation of IMS and IMS/MS/MS Findings}

A general conclusion drawn from the studies described above was that the degree of solvation by ketone of protonated ammonia and the protonated hydrazines decreased as the number of methyl groups increased.

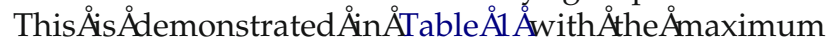
number of ketones observed on ions with reagent gas in either the source region only or in the entire drift tube. The number of adduct molecules is at a maximum when ketone is present throughout the whole drift tube and the maximum values are: 4 for $\mathrm{NH}_{4}^{+}, 4$ or 3 for $\mathrm{HZ} \cdot \mathrm{H}^{+}, 3$ or 2 for $\mathrm{MMH} \cdot \mathrm{H}^{+}$, and 2 or 1 for each of $\mathrm{UDMH} \cdot \mathrm{H}^{+}$and $\mathrm{TMH} \cdot \mathrm{H}^{+}$. A ketone will be most strongly hydrogenbonded at sites with the highest charge density. Protonation of MMH and UDMH will occur at the alkylated nitrogen where inductive forces stabilize the charge. An $\mathrm{ab}$ initio calculation of protonated $\mathrm{MMH}$ at the HF 6-31G* level showed that protonation on the alkylated nitrogen was favored by $4.7 \mathrm{kcal} \mathrm{mol}^{-1}$ and natural bond order (NBO) analysis showed that $84 \%$ of the positive charge resided on the $-\mathrm{N}\left(\mathrm{CH}_{3}\right) \mathrm{H}_{2}$ end of the molecule. The charge on the hydrogens attached to this $\mathrm{N}$ ensures that they are the favored sites for association with the oxygen of a ketone. For the ions of interest there are four acidic hydrogens on $\mathrm{NH}_{4}^{+}$, three on $\mathrm{H}_{2} \mathrm{NNH}_{3}^{+}$, two on $\mathrm{H}_{2} \mathrm{NN}(\mathrm{Me}) \mathrm{H}_{2}^{+}$, and one on each of $\mathrm{H}_{2} \mathrm{NN}(\mathrm{Me})_{2} \mathrm{H}^{+}$and $\mathrm{H}_{2} \mathrm{NN}(\mathrm{Me})_{2} \mathrm{H}^{+}$. This is in general agreement Âvith Åhe

When ketone is present throughout a drift tube, 
ammonium attaches a maximum of four ketone molecules but only two are observed when the ketone is present only in the source. This shows that ion-

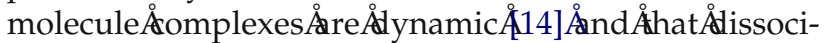
ation of ligands will occur in the absence of ketone in the drift gas. An HPMS study gave thermodynamic data for the association of acetone with the ammonium ion $\AA 15]$. ÅThe Åfirst Åand Åsecond ÅketoneÅmolecules Åare bound more strongly than the third and fourth while the fifth, which must be in an outer shell, is much more weakly bound. Use of the thermodynamic data shows that ammonium solvated by four ketone molecules is the predominant ion in the system at equilibrium over the acetone concentration range $10^{-4}$ to $10^{-2} \mathrm{ppm}_{\mathrm{v}}$. Thermodynamic data are not available for other ketones or for the association of ketones with hydrazines, but a similar argument must hold regarding stability of the complexes in the presence and absence of ketone in the drift gas.

The formation of hydrazones and azines in this work is inconsistent with the IMS/MS/MS results reported

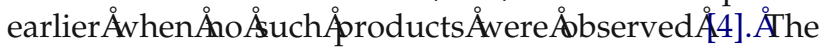
major difference was in the mobility spectrometer where the IMS/MS instrument was based on a ceramic drift tube. In contrast, the tube in the prior report was made with gold-plated drift rings and a Teflon source region in a stainless steel housing. Surface-induced condensation reactions on the ceramic between hydrazine and ketones could have led to neutral hydrazones and azines, which were then protonated. Since hydrazone is known to form in an acid-catalyzed reaction in

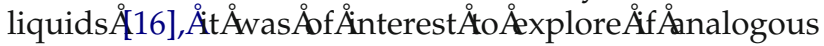
gas-phase reactions could be observed in a mass spectrometry with extended mass range, and CID experiments were used to search for possible hydrazone and azine formation initiated by collisional activation of such complexes. Studies by APCI-MS were also completed to determine the maximum number of 5-nonanone molecules that readily, that is strongly, attach to protonated ammonia and to each of the protonated hydrazines, with positive identification of the complexes.

\section{Studies with Atmospheric Pressure Chemical Ionization Mass Spectrometry. Sciex API-III}

In the absence of ammonia or one of the hydrazines, the APCI spectrum contained only the usual hydrated proton peaks, $\left(\mathrm{H}_{2} \mathrm{O}\right)_{n} \mathrm{H}^{+}$, obtained with ambient air; $n$ was 1 through 4 . The identities of these complexes and all others of interest in the study were confirmed by CID. The CID spectra for $\left(\mathrm{H}_{2} \mathrm{O}\right)_{n} \mathrm{H}^{+}$contained solely product ions resulting from sequential loss of $\mathrm{H}_{2} \mathrm{O}$. Since the proton affinity of ammonia is far higher than that of water, the $\left(\mathrm{H}_{2} \mathrm{O}\right)_{n} \mathrm{H}^{+}$ions were replaced by $\left(\mathrm{NH}_{3}\right)_{\mathrm{m}} \mathrm{H}^{+}$and $\left(\mathrm{NH}_{3}\right)_{\mathrm{m}} \mathrm{H}^{+}\left(\mathrm{H}_{2} \mathrm{O}\right)_{\mathrm{n}}$ when ammonia was contained in the air. The relative intensities of the two sets of peaks, $\left(\mathrm{NH}_{3}\right)_{\mathrm{m}} \mathrm{H}^{+}$and $\left(\mathrm{NH}_{3}\right)_{\mathrm{m}} \mathrm{H}^{+}\left(\mathrm{H}_{2} \mathrm{O}\right)_{\mathrm{n}}$, depended on the ammonia concentration. The APCI-MS spectra obtained with each of the hydrazines was similar, with major peaks due to $(\mathrm{M})_{\mathrm{m}} \mathrm{H}^{+}$and $(\mathrm{M})_{\mathrm{m}} \mathrm{H}^{+}\left(\mathrm{H}_{2} \mathrm{O}\right)_{\mathrm{n}}$ $(\mathrm{M}=\mathrm{HZ}, \mathrm{MMH}$, or UDMH). A typical spectrum for hydrazine showed $100 \%$ relative abundance for $\mathrm{HZH}^{+}$, $52 \%$ for $\mathrm{HZH}^{+} \mathrm{H}_{2} \mathrm{O}$, and $35 \%$ for $(\mathrm{HZ})_{2} \mathrm{H}^{+}$. A very small peak was observed at $\mathrm{m} / \mathrm{z} 83$ corresponding to the hydrated dimer, $(\mathrm{HZ})_{2} \mathrm{H}^{+}\left(\mathrm{H}_{2} \mathrm{O}\right)$. Higher concentrations of hydrazine produced the higher cluster $(\mathrm{HZ})_{3} \mathrm{H}^{+}$. The CID spectrum of this ion showed sequential evaporation of neutral hydrazine leading to $(\mathrm{HZ}) \mathrm{H}^{+}$in agree-

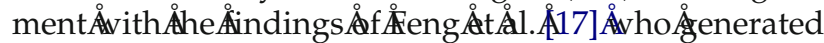
$(\mathrm{HZ})_{\mathrm{n}} \mathrm{H}^{+}$by electron impact in an ion-drift source and obtained CID spectra by scanning the electrostatic analyzer voltage of a double focusing instrument.

Sufficient 5-nonanone was introduced into the ionization region so that all the $(\mathrm{HZ})_{\mathrm{n}} \mathrm{H}^{+}$and its hydrates disappeared, and new ions, identified by CID as protonated hydrazine solvated by one, two, and three 5-nonanone molecules, $(\mathrm{HZ}) \mathrm{H}^{+}$(non), $(\mathrm{HZ}) \mathrm{H}^{+}$(non) $)_{2}$ and $(\mathrm{HZ}) \mathrm{H}^{+}$(non) $)_{3}$, appeared and were formed through reactions in eq 10 :

$$
(\mathrm{HZ}) \mathrm{H}^{+}\left(\mathrm{H}_{2} \mathrm{O}\right)_{\mathrm{n}}+\mathrm{x}(\text { non }) \rightarrow(\mathrm{HZ}) \mathrm{H}^{+}(\text {non })_{\mathrm{x}}
$$

To facilitate observation of any higher solvates, the concentration of 5-nonanone in the inlet flow was increased further and the flow of plenum gas was reduced to a minimum to preserve, as much as possible, vapor concentrations of 5-nonanone in the interface region. These measures did not lead to the appearance in the spectrum of $(\mathrm{HZ}) \mathrm{H}^{+}$(non) $)_{4}$ or higher solvates. The implication is that if such complexes were formed, any 5-nonanone beyond the third was so weakly bound relative to the first three that it was easily removed in passage through the interface region of this mass spectrometer. This conclusion is in accord with the observation $\AA$ f for $n=4$, a so-called "magic number", for the series $(\mathrm{HZ})_{n} \mathrm{H}^{+}$. Computation for $\mathrm{H}_{2} \mathrm{NNH}_{3}^{+}$showed that the three hydrogens on the nitrogen protonation site are all

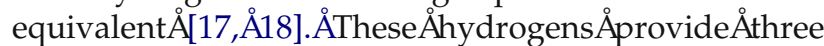
equivalent sites for strongly binding a maximum of 3 bases, three $\mathrm{HZ}$ in $(\mathrm{HZ})_{4} \mathrm{H}^{+}$and three 5-nonanone molecules in the case of $(\mathrm{HZ}) \mathrm{H}^{+}$(non) $)_{3}$. Because of steric hindrance and lack of a suitable site for hydrogen bonding, further association of 5-nonanone with $(\mathrm{HZ}) \mathrm{H}^{+}$(non) $)_{3}$ would have to occur at the $\mathrm{NH}_{2}$ end, which does not bear the charge. Steric considerations and lack of suitable hydrogen bonding sites at the crowded $\mathrm{NH}_{3}$ (non) $)_{3}$ end of the complex would preclude the possibility of a fourth 5-nonanone in an outer shell.

The CID spectrum of $(\mathrm{HZ}) \mathrm{H}^{+}$(non) $)_{3}$ with ion energy of $60 \mathrm{eV}$ gave the major fragment ions $(\mathrm{HZ}) \mathrm{H}^{+}$(non) $)_{2}$,

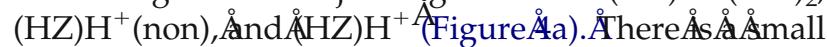
peak, constituting $<3 \%$ of the total product ion intensity, due to (non) $\mathrm{H}^{+}$and there is a trace of (non) ${ }_{2} \mathrm{H}^{+}$. No (non) ${ }_{3} \mathrm{H}^{+}$was observed at any collision energy. The CID spectrum of $(\mathrm{HZ}) \mathrm{H}^{+}$(non) $)_{2}$ shown $\AA$ in $\AA$ Figure $\AA$ ab 


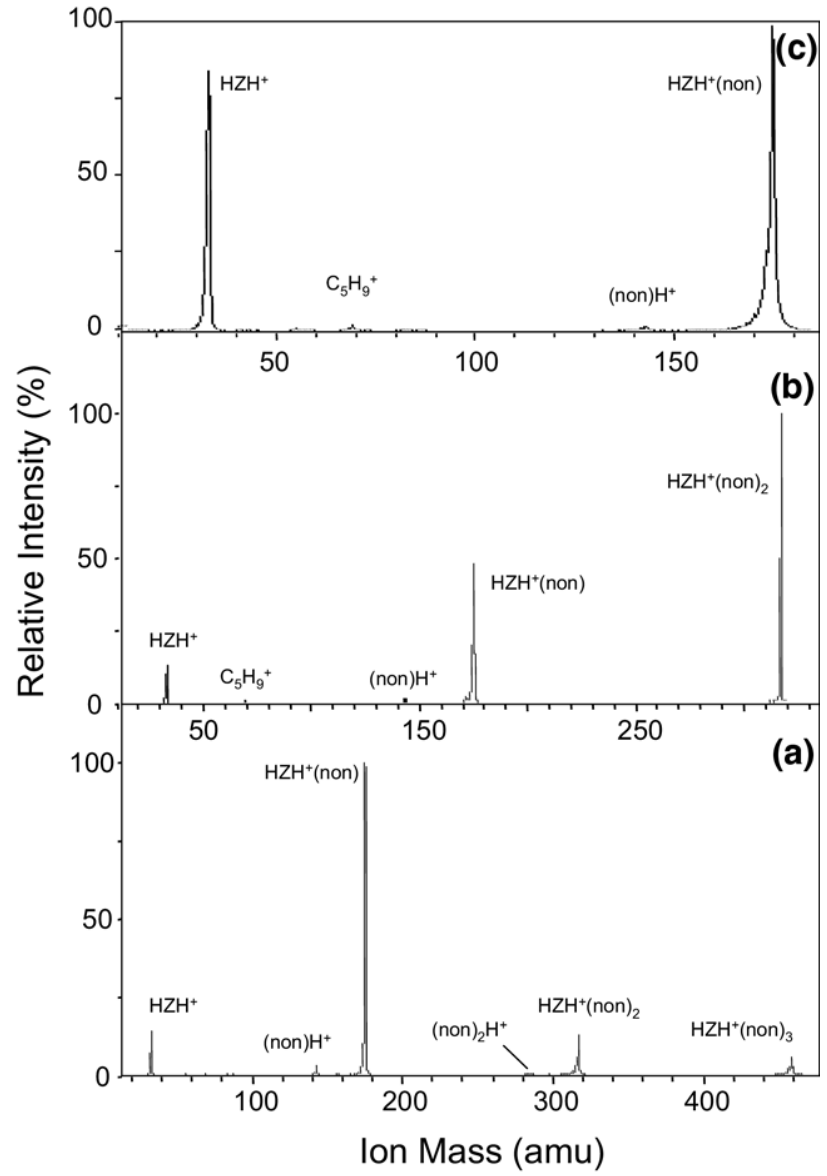

Figure 4. CID spectra from APCI-MS of ion-molecule complexes: (a) $(\mathrm{HZ}) \mathrm{H}^{+}(\text {non })_{3}, 55 \mathrm{eV}$ ion energy; (b) $\left(\mathrm{HZ} \mathrm{H}^{+} \text {(non) }\right)_{2}, 40 \mathrm{eV}$ ion energy; (c) $(\mathrm{HZ}) \mathrm{H}^{+}($non), $50 \mathrm{eV}$ ion energy.

contained $(\mathrm{HZ}) \mathrm{H}^{+}$(non) and $(\mathrm{HZ}) \mathrm{H}^{+}$as the major products. No (non) ${ }_{2} \mathrm{H}^{+}$is observable but (non) $\mathrm{H}^{+}$is present, constituting $<2 \%$ of the products. The CID spectrum of the low intensity $(\mathrm{HZ}) \mathrm{H}^{+}$(non) ion showed that the

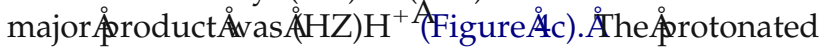
ketone, (non) $\mathrm{H}^{+}$, was present to the extent of less than $2 \%$. This distribution of products from the dissociation of the proton-bound mixed dimer is surprising, considering that the evaluated proton affinities of hydrazine $\left(853.2 \mathrm{~kJ} \mathrm{~mol}^{-1}\right)$ and 5-nonanone $(853.7 \mathrm{~kJ}$ $\mathrm{mol}^{-1}$ )ÅareÅessentially ÅidenticalÅ[19]. ÅTheÅevaluated basicities are also identical so there is apparently no entropy effect that would favor $(\mathrm{HZ}) \mathrm{H}^{+}$(non) as the overwhelming product.

The APCI-MS spectrum of ammonia in the presence of excess 5-nonane contained the ions with intensities in the order: $\mathrm{NH}_{4}^{+}$(non) $\ll \mathrm{NH}_{4}^{+}(\text {non })_{2}<\mathrm{NH}_{4}^{+}(\text {non })_{4}<$ $\mathrm{NH}_{4}^{+}$(non) $)_{3}$. Attempts to observe $\mathrm{NH}_{4}^{+}(\text {non })_{5}$ and higher solvates by minimizing both the plenum gas flow rate and the declustering potentials of the interface and focusing assembly were unsuccessful. In these experiments, the intensity of $\mathrm{NH}_{4}^{+}(\text {non) })_{4}$ relative to those of the lower solvates could be increased but was attributed more by the appearance of $\mathrm{NH}_{4}^{+}(\text {non })_{2}\left(\mathrm{H}_{2} \mathrm{O}\right)_{\mathrm{n}}$
( $n=1$ to 4$)$ and $\mathrm{NH}_{4}^{+}(\text {non })_{3}\left(\mathrm{H}_{2} \mathrm{O}\right)_{n}(n=1$ to 8$)$ than by an increase in the absolute intensity of $\mathrm{NH}_{4}^{+}$(non) $)_{4}$. The CID spectra of $\mathrm{NH}_{4}^{+}$(non) $)_{\mathrm{n}}$ were dominated by the sequential loss of 5-nonanone and, as was observed in the CID spectra of $(\mathrm{HZ}) \mathrm{H}^{+}$(non) $)_{\mathrm{n}}$, the peaks due to $\mathrm{H}^{+}$(non) and $\mathrm{H}^{+}$(non) $)_{2}$ constituted less than $2 \%$ of total ion intensity. The core ion in all the products remained $\mathrm{NH}_{4}^{+}$even though the proton affinity of ammonia (853.6 $\mathrm{kJ} \mathrm{mol}^{-1}$ ) is identical to that of 5-nonanone.

Monomethyl hydrazine was introduced into the ion source together with a concentration of 5-nonanone, such that all the $(\mathrm{MMH})_{\mathrm{n}} \mathrm{H}^{+}$had disappeared. The major peak was then $(\mathrm{MMH}) \mathrm{H}^{+}(\text {non })_{2}$ together with a small amount of $(\mathrm{MMH}) \mathrm{H}^{+}$(non). There was no evidence for the formation of $(\mathrm{MMH}) \mathrm{H}^{+}$(non) $)_{3}$. As shown

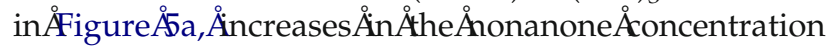
resulted in the appearance of a very small peak at $\mathrm{m} / \mathrm{z}$ $473,(\mathrm{MMH}) \mathrm{H}^{+}$(non) $)_{3}$, accompanied by one of approximately equal intensity at $\mathrm{m} / \mathrm{z} 445$ that was identified as (non) ${ }_{3} \mathrm{H}^{+}\left(\mathrm{H}_{2} \mathrm{O}\right)$. By analogy with results obtained from a high-pressure mass spectrometric and computational

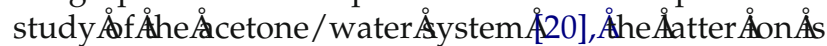
probably a central $\mathrm{H}_{3} \mathrm{O}^{+}$with three hydrogen bonds to the 5-nonanone neutrals. When the flow of 5-nonanone was increased further and the plenum gas flow was minimized, hydration of $(\mathrm{MMH}) \mathrm{H}^{+}(\text {non })_{2}$ rather than increased formation of $(\mathrm{MMH}) \mathrm{H}^{+}(\text {non })_{3}$ was observed. There was no $(\mathrm{MMH}) \mathrm{H}^{+}(\text {non })_{4}$ in the spectrum. The CID spectrum of $(\mathrm{MMH}) \mathrm{H}^{+}(\text {non })_{2}$ at an ion energy of $47 \mathrm{eV}$ contained only two products, $(\mathrm{MMH}) \mathrm{H}^{+}$(non)

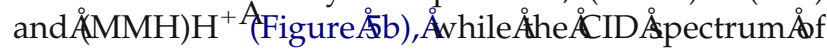
$(\mathrm{MMH}) \mathrm{H}^{+}$(non) at ion energies up to $80 \mathrm{eV}$ contained

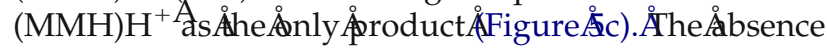
of any trace of (non) ${ }_{n} \mathrm{H}^{+}$in the spectra is in accord with the observations for the hydrazine complexes but this time is to be expected because of the higher proton affinity of MMH $\left(898.8 \mathrm{~kJ} \mathrm{~mol}^{-1}\right)$ compared with that of 5-nonanone (853.7 kJ mol$\left.{ }^{-1}\right)$.

In the absence of 5-nonanone, UDMH was protonated to give mainly $(\mathrm{UDMH}) \mathrm{H}^{+}$and $(\mathrm{UDMH})_{2} \mathrm{H}^{+}$. When 5-nonanone was introduced the major peak was $(\mathrm{UDMH}) \mathrm{H}^{+}$(non), but a small amount of $(\mathrm{UDMH}) \mathrm{H}^{+}$(non) $)_{2}$ was also formed. At a relatively high concentration of 5-nonanone, the proton-bound dimer of the ketone was formed rather than more $(\mathrm{UDMH}) \mathrm{H}^{+}(\text {non })_{2}$ as Åshown ̊̊n $\mathrm{Figure} \AA_{6}$ a. ÅAt Åhigher 5-nonanone concentration, both $(\mathrm{UDMH}) \mathrm{H}^{+}$(non) ${ }_{2}$ and (non) ${ }_{2} \mathrm{H}^{+}$disappeared as a peak at $\mathrm{m} / \mathrm{z} 331$ appeared $\AA$ (Figure $\AA 6$ b b). ÅThis Åpeak $A$ was Aidentified $\AA_{b y}$ $\mathrm{CID}$ as $(\mathrm{MMH}) \mathrm{H}^{+}$(non) $)_{2}$. The source of the $\mathrm{MMH}$ is unknown, but it is most probably an impurity in the UDMH, even though GC-FID and GC-MS analyses of the UDMH did not show measurable levels of contamination. The CID spectra showed that qualitatively the second 5-nonanone of $(\mathrm{UDMH}) \mathrm{H}^{+}$(non) $)_{2}$ was very weakly bound compared with the first, and the only major products in the spectrum were $(\mathrm{UDMH}) \mathrm{H}^{+}$(non) and $(\mathrm{UDMH}) \mathrm{H}^{+}$. The only product in the CID spectrum of $(\mathrm{UDMH}) \mathrm{H}^{+}$(non) was (UD- 


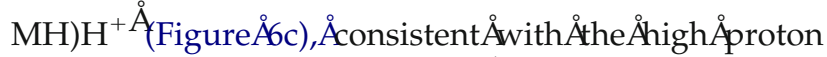
affinity of UDMH, $927.1 \mathrm{~kJ} \mathrm{~mol}^{-1}$, relative to that of the ketone.

The APCI-MS results agree with those from the IMS/MS study. A protonated hydrazine or the ammonium ion will effectively bind 5-nonanone molecules in a number equal to the number of hydrogens on the protonated nitrogen. Because methyl substitution at a nitrogen leads to increased basicity of that nitrogen, the expected number of strongly bound ketones is four for ammonia; three for $\mathrm{HZ}$, two for $\mathrm{MMH}$, and one for UDMH. The exception is UDMH, which appears to be able to attach a second ketone rather weakly but with sufficient strength to traverse the mass spectrometer curtain gas. The significant and troublesome difference between the IMS/MS and the APCI-MS results was the absence of hydrazone formation in the latter; further investigation by APCI-MS was warranted.

\section{Study of Gas Phase Formation of Hydrazone and Azine by CID Experiments}

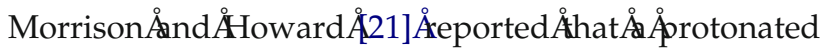
hydrazone is observed under chemical ionization con-

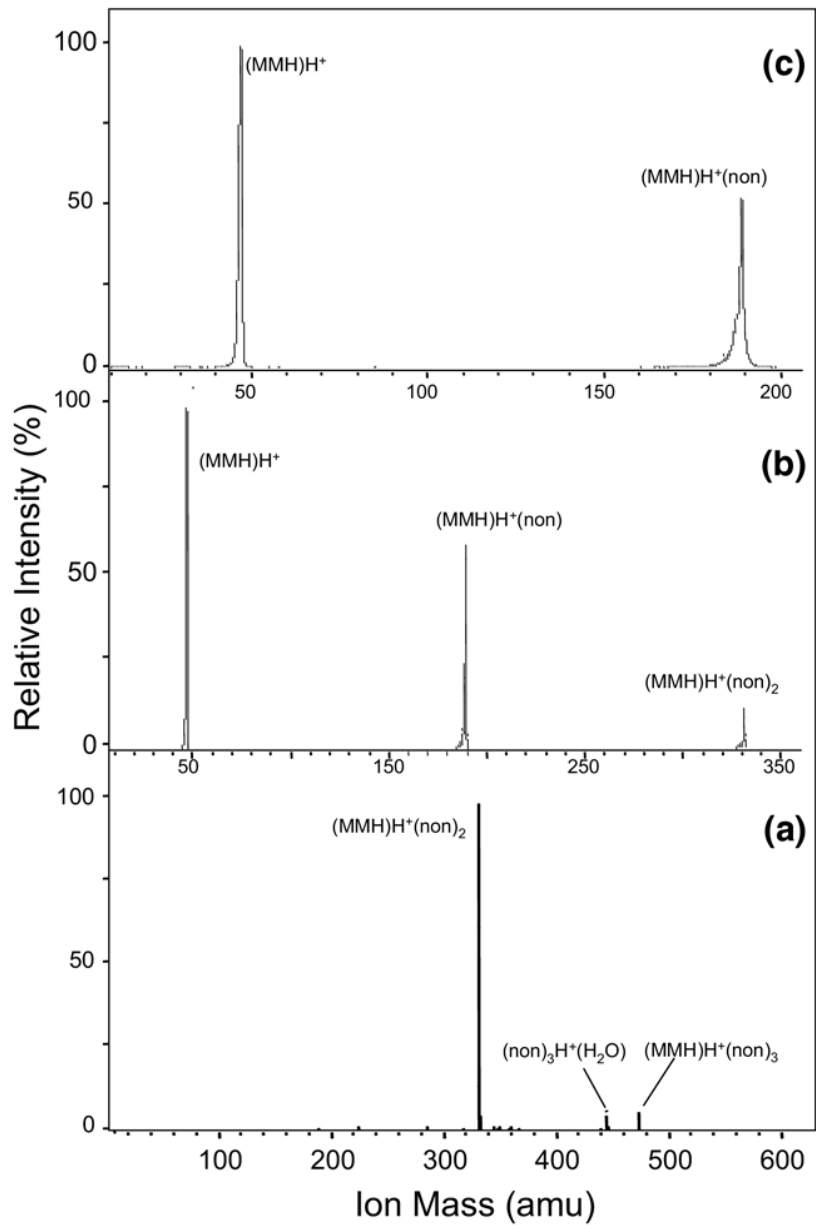

Figure 5. Spectra from APCI-MS for MMH with 5-nonanone (a), CID spectra of $(\mathrm{MMH}) \mathrm{H}^{+}(\text {non })_{2}, 45 \mathrm{eV}$ ion energy $(\mathbf{b})$, and at $55 \mathrm{eV}$ ion energy (c).

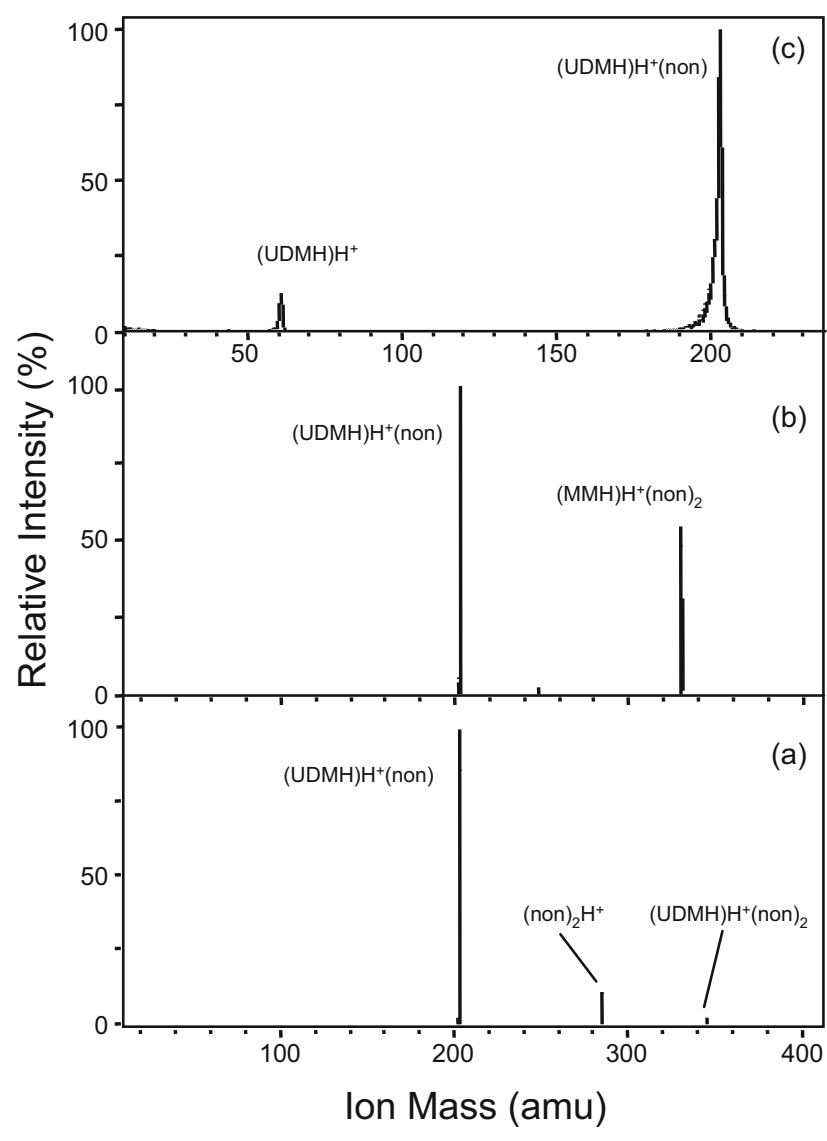

Figure 6. Spectra from APCI-MS for 1,1-dimethylhydrazine with 5-nonanone (a), for UDMH and increased ketone vapor concentration (b), and the CID spectrum for $(\mathrm{UDMH}) \mathrm{H}^{+}$(non) at $90 \mathrm{eV}$ ion energy (c).

ditions in a drift tube attached to a mass spectrometer in the reaction of $(\mathrm{HZ}) \mathrm{H}^{+}$with aldehydes and ketones (eq 11).

$$
\mathrm{R}_{1} \mathrm{R}_{2} \mathrm{CO}+\mathrm{N}_{2} \mathrm{H}_{5}^{+} \rightarrow \mathrm{R}_{1} \mathrm{R}_{2} \mathrm{CN}_{2} \mathrm{H}_{3}^{+}+\mathrm{H}_{2} \mathrm{O}
$$

The drift gas was helium at 1.4 torr. Formaldehyde, acetaldehyde, propanal, octanal, and nonanal all gave the protonated hydrazone as the sole product. The ketones, acetone, 2-butanone, pentanones, and 3-hexanone, also produced the protonated hydrazone though favored formation of the ion-ketone complex (eq 12):

$$
\mathrm{R}_{1} \mathrm{R}_{2} \mathrm{CO}+\mathrm{N}_{2} \mathrm{H}_{5}^{+} \rightarrow\left(\mathrm{R}_{1} \mathrm{R}_{2} \mathrm{CO}\right) \mathrm{H}^{+}\left(\mathrm{N}_{2} \mathrm{H}_{4}\right)
$$

A later paper describes a further, more detailed inves-

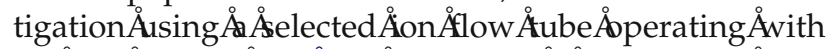

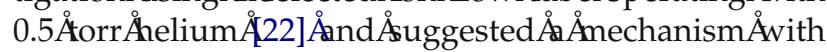
low activation energy involving the association of a second hydrazine with an initially formed $\mathrm{R}_{1} \mathrm{R}_{2} \mathrm{CON}_{2} \mathrm{H}_{5}^{+}$. The resulting complex rearranges to form the protonated hydrazone, water, and hydrazine. It was also reported that the reaction rates for hydrazone formation were enhanced by translational activation in the drift field (eq 13): 
Table 2. Drift times and cross sections for 5-nonanone complexes with ammonia and hydrazines in supporting atmosphere of air

\begin{tabular}{lcccccrr}
\hline & Time & $\mathrm{d}$ & $\Omega$ & lon mass & $\mu$ & Sqrt $\mu$ & $1 /\left(\mu^{1 / 2} \Omega\right)$ \\
\hline \hline & $\mathrm{ms}$ & $\AA$ & $\AA^{2}$ & $\mathrm{Da}$ & $\mathrm{Da}$ & & \\
Ammonia & 25.45 & 15.73 & 247 & 586 & 27.45 & 5.239 & 0.000186 \\
$\mathrm{HZ}$ & 21.89 & 14.78 & 221 & 459 & 27.10 & 5.206 & 0.000245 \\
MMH & 18.43 & 13.34 & 185 & 331 & 26.50 & 5.147 & 0.000357 \\
UDMH & 18.3 & 11.87 & 151 & 203 & 25.22 & 5.022 & 0.000454 \\
\hline
\end{tabular}

$$
\mathrm{R}_{1} \mathrm{R}_{2} \mathrm{CO}\left(\mathrm{N}_{2} \mathrm{H}_{4}\right)_{2} \mathrm{H}^{+} \rightarrow \mathrm{R}_{1} \mathrm{R}_{2} \mathrm{CN}_{2} \mathrm{H}_{3}^{+}+\mathrm{H}_{2} \mathrm{O}+\mathrm{N}_{2} \mathrm{H}_{4}
$$

A protonated hydrazone formed from protonated hydrazine solvated by 5 -nonanone would have the formula $\mathrm{H}_{2} \mathrm{~N}_{2}\left(\mathrm{H}^{+}\right) \mathrm{C}_{9} \mathrm{H}_{18}$ (non) $)_{n}$, where $n$ is the number of solvating ketone molecules retained in the desolvation reaction as in eq 14:

$$
\begin{gathered}
\left(\mathrm{N}_{2} \mathrm{H}_{4}\right) \mathrm{H}^{+}(\text {non })_{\mathrm{n}+\mathrm{x}} \rightarrow \mathrm{H}_{2} \mathrm{~N}_{2}\left(\mathrm{H}^{+}\right) \mathrm{C}_{9} \mathrm{H}_{18}(\text { non })_{\mathrm{n}}+\mathrm{H}_{2} \mathrm{O} \\
+(\mathrm{x}-1)(\text { non })
\end{gathered}
$$

Since no $\mathrm{H}_{2} \mathrm{~N}_{2}\left(\mathrm{H}^{+}\right) \mathrm{C}_{9} \mathrm{H}_{18}$ (non) $)_{\mathrm{n}}$ or similar ions from other hydrazines were observed in any of the APCI or APCI/CID spectra, it must be concluded that hydrazones are not formed as products of the collisional activation of protonated hydrazines solvated by one or a few 5-nonanone molecules.

The possibility of hydrazone formation via protonbound complexes containing two hydrazine molecules was also investigated. The CID spectra at $50 \mathrm{eV}$ for $(\mathrm{HZ})_{2} \mathrm{H}^{+}$(non) and $(\mathrm{MMH})_{2} \mathrm{H}^{+}$(non) show that the complexes competitively lose hydrazine and ketone as exemplified by eq 15 for $(\mathrm{HZ})_{2} \mathrm{H}^{+}$(non).

$$
\begin{array}{ccc} 
& & (\mathrm{HZ})_{2} \mathrm{H}^{+}+\text {non } \\
& \nearrow & \\
& \searrow & \\
& & \left.(\mathrm{HZ})_{2} \mathrm{H}^{+} \text {(non) } \mathrm{H}^{+} \text {(non }\right)+\mathrm{Hz}
\end{array}
$$

Protonated hydrazone would appear at $m / z \quad 189$, $\mathrm{H}_{2} \mathrm{~N}_{2}\left(\mathrm{H}^{+}\right) \mathrm{C}_{9} \mathrm{H}_{18}(\mathrm{HZ})^{+}$, if the second hydrazine were retained, or at $m / z 157, \mathrm{H}_{2} \mathrm{~N}_{2}\left(\mathrm{H}^{+}\right) \mathrm{C}_{9} \mathrm{H}_{18}$, if it were not. No such ions are present. There was no evidence for the formation of protonated hydrazone from $(\mathrm{MMH})_{2} \mathrm{H}^{+}$(non) at $\mathrm{m} / \mathrm{z}$ 217, $\left[\mathrm{H}_{2} \mathrm{~N}\left(\mathrm{CH}_{3}\right) \mathrm{N}\left(\mathrm{H}^{+}\right) \mathrm{C}_{9} \mathrm{H}_{18}(\mathrm{MMH})\right]^{+}$, or at $m / z 171$, $\mathrm{H}_{2} \mathrm{~N}\left(\mathrm{CH}_{3}\right) \mathrm{N}\left(\mathrm{H}^{+}\right) \mathrm{C}_{9} \mathrm{H}_{18}$. Hydrazone is not formed by collisional activation of the simple ion-molecule complexes formed by APCI in the manner described. The formation must occur by a different and probably more complex mechanism than that described by Morrison and Åcoworkers $\AA 22]$. Ån Åconclusion, $\AA$ Ahere $\AA$ is $\AA$ no $\AA$ strong evidence in these studies that the hydrazones were formed from gas-phase reactions of ions and the production of neutral hydrazones and hydrazines by a surface reaction in our IMS/MS study cannot be discarded.

\section{Calculations of Ion Size and Mobility}

The model of a ion-molecule complex with ketone molecules equal in number to the hydrogens on the protonated nitrogen, four for $\mathrm{NH}_{4}^{+}$, three for $\mathrm{NH}_{2} \mathrm{NH}_{3}^{+}$, two for $\mathrm{NH}_{2} \mathrm{~N}\left(\mathrm{CH}_{3}\right) \mathrm{H}_{2}^{+}$, and one for $\mathrm{NH}_{2} \mathrm{~N}\left(\mathrm{CH}_{3}\right)_{2} \mathrm{H}^{+}$ can be seen in a linear correlation of ion mass and drift time. The expression for the drift time $t_{d}$ of an ion in the

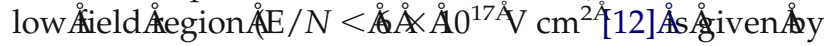
eq 16:

$$
\mathrm{t}_{\mathrm{d}}=\frac{\mathrm{E}}{1} \frac{3}{16} \frac{\mathrm{q}}{\mathrm{N}}\left(\frac{2 \pi}{\mu \mathrm{kT}}\right)^{\frac{1}{2}} \frac{(1+\alpha)}{\Omega(\mathrm{T})}
$$

where terms are E, the electric field strength; 1, the drift length; $\mathrm{N}$, the number density of the drift gas; $\mathrm{q}$, the electronic charge; $\mu$, the reduced mass of the colliding ion-neutral pair; and $\alpha$, a small factor that is almost zero and Ås Årually Ågnored $\AA$ 12]. ̊̊he Årm $\AA$ (T) momentum-transfer cross section for the collision between ion and drift gas molecule, i.e., an average ion-neutral collision cross section.

If the hard sphere model is taken as an approximation of the ion-neutral interaction, then $\Omega=\pi \mathrm{d}^{2}$ where

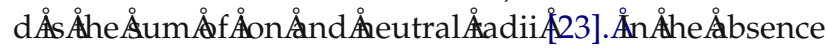
of an intimate knowledge of the collision cross section, a crude approximation for ion size is a freely rotating body with a cross section of area $\pi \mathrm{r}^{2}$, where the radius $r$ is equal to one half the largest linear dimension. The diameters of the ions computed by molecular mechanics were $15.73 \AA$ for $\mathrm{NH}_{4}(\text { non })_{4}^{+}, 14.78 \AA$ for $(\mathrm{HZ}) \mathrm{H}^{+}(\text {non })_{3}, 13.34 \AA$ for $(\mathrm{MMH}) \mathrm{H}^{+}$(non) $)_{2}^{+}$, and 11.87 $\AA$ for $(\mathrm{UDMH}) \mathrm{H}^{+}$(non) $\mathrm{H}^{+}$; the respective ion-neutral cross sections are, respectively, $247 \AA^{2}, 221 \AA^{2}, 185 \AA^{2}$, $151 \AA^{2}$ for a average diameter of $2 \AA$ for nitrogen and

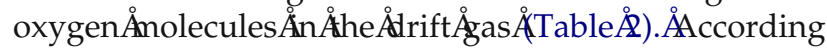
to eq $16, t_{d}$ for a series of similar ions should be a linear function the reciprocal of $\left(\Omega \mu^{1 / 2}\right)$ under constant experimental conditions. If the identification of ions above are correct in the IMS spectra, then a plot of $1 /\left(\Omega \mu^{1 / 2}\right)$ should generate a straight line. This was obtained for the first three ions following the formula $1 / \Omega(\mu)^{1 / 2}=$ $-2.43 \times 10^{-5} \pm 0.484 \times 10^{-5}-7.96 \times 10^{-4} \pm 1.01 \times$ $10^{-4}$ with an $r^{2}$ of 0.965 . However, the drift time for the UDMH complex, $\mathrm{NH}_{2} \mathrm{~N}\left(\mathrm{CH}_{3}\right)_{2}$ (non) $\mathrm{H}^{+}$, is too large compared with the expected value and is almost identical with that of the $\mathrm{MMH}$ complex, $\mathrm{NH}_{2} \mathrm{~N}\left(\mathrm{CH}_{3}\right) \mathrm{H}_{2}$ (non) $)_{2}^{+}$. There are three possibilities for this anomalous behavior: (1) the major UDMH complex in the drift tube contains two ketone molecules rather 
than one, as suggested; (2) $\mathrm{MMH}$ is present as an impurity in the UDMH; and (3) MMH or its protonated form is produced in an ion-molecule reaction in the ion source of the mobility spectrometer. The APCI results would seem to negate possibility (3) but there is not sufficient information to distinguish between the possibilities (1) and (2).

\section{Conclusions}

Drift times for ions of ammonia and hydrazines in a supporting atmosphere containing ketone vapor are seemingly anomalous from masses and sizes of protonated molecules alone and can be understood instead as ion-molecule complexes. The number of ketones attached to the protonated molecule agreed with an ion structure equal to the number of protons available for associations, the drift times, and mass-analysis. The implication for IMS and IMS/MS experiments is that ions with comparable mobility can be separated when gas atmospheres are modified with a reagent gas. In this, the core ion is not chemically altered but the overall ion mobility is changed. This was attained in all instances with several drift tubes for highly reactive hydrazines and might be recommended for other IMS/MS studies where improvements are needed in separating core ions by mobility. The reagent gas giving best separation and detection of ammonia and hydrazines underẢaboratory

\section{Acknowledgments}

The authors thank Dr. G. C. Morrison for a preprint of the paper.

\section{References}

1. Taffe, P. A.; Rose-Pehrsson, S. L. Colorimetric Method for Real-Time Detection of Hydrazine and Its Derivatives. U.S.A. patent, 1990; 4900681 A, 19900213.

2. Wang, J.; Chatrathi, M. P.; Tian, B.; Polsky, R. Capillary Electrophoresis Chips with Thick-Film Amperometric Detectors: Separation and Detection of Hydrazine Compounds. Electroanalysis 2000, 12, 691-94.
3. Leasure, C. S.; Eiceman, G. A. Continuous Detection of Hydrazine and Monomethylhydrazine Using Ion Mobility Spectrometry. Anal. Chem. 1985, 57, 1890-1894.

4. Eiceman, G. A.; Salazar, M. R.; Rodriguez, M. R.; Limero, T. F.; Beck, S. W.; Cross, J. H.; Young, R.; James. J. T. Ion Mobility Spectrometry of Hydrazine, Monomethylhydrazine, and Ammonia in Air with 5-Nonanone Reagent Gas. Anal. Chem. 1993, 65, 1696-1702.

5. Eiceman, G. A.; Nazarov, E. G.; Stone, J. A.; Rodriguez, J. E. Analysis of a Drift Tube At Ambient Pressure: Models and Precise Measurements in Ion Mobility Spectrometry. Rev. Sci. Instrum. 2001, 72, 3610-3621.

6. Kim, S. H.; Betty, K. R.; Karasek, F. W. Mobility Behavior and Composition of Hydrated Positive Reactant Ions in Plasma Chromatography with Nitrogen Carrier Gas. Anal. Chem. 1978, 50, 2006-2012.

7. Eiceman, G. A.; Karpas, Z. Ion Mobility Spectrometry. CRC Press: Boca Raton, 1994, pp 80-84.

8. Sunner, J.; Nicol, G.; Kebarle, P. Factors Determining Relative Sensitivity of Analytes in Positive Mode Atmospheric Pressure Ionization Mass Spectrometry. Anal. Chem. 1988, 60, 1300-1307.

9. Watts, P. Use of Ion Mobility Spectroscopy for the Detection and Analysis of Vapors. Anal. Proc. 1991, 28, 328-331.

10. Ewing, R. G.; Eiceman, G. A.; Stone, J. A. Proton-Bound Cluster Ions in Ion Mobility Spectrometry. Int. J. Mass Spectrom. 1999, 193, 57-68.

11. Eiceman, G. A.; Wang, Y. Detection of Microorganisms and Related Chemical Compounds Using Ion Mobility Spectrometry, Final Report on Prime Contract DAAA15-90-C-1006, Subcontract GC-2192-91-002: August 10, 1993; New Mexico State University, Las Cruces, NM.

12. Revercomb, H. E.; Mason, E. A. Theory of Plasma Chromatography Gaseous Electrophoresis-A Review. Anal. Chem. 1975, 47, 970-983.

13. Karpas, Z. Ion Mobility Spectrometry of Aliphatic and Aromatic Amines. Anal. Chem. 1989, 61, 684-689.

14. Preston, J. M.; Rajadhyax, L. Effect of Ion/Molecule Reactions on Ion Mobilities. Anal. Chem. 1988, 60, 31-34.

15. Meot-Ner, M.; Sieck, L.W.; Liebman, J. F.; Scheiner, S. Complexing of the Ammonium Ion by Polyethers. Comparative Complexing Thermochemistry of Ammonium, Hydronium, and Alkali Cations. J. Phys. Chem. 1996, 100, 6445-6450.

16. Fessenden, R. J.; Fessenden, J. S. Organic Chemistry, Willard Grant Press: Boston, 1979, pp 528-529.

17. Feng, W. Y.; Aviyente, V.; Varnali, T.; Lifshitz, C. Structures, Energetics, and Reactions of Proton-Bound Hydrazine Clusters. J. Phys. Chem. 1995, 99, 1776-1785.

18. Gill, P. M. W.; Radom, L. Structures and Stabilities of the Dimer Dications of First- and Second-Row Hydrides. J. Am. Chem. Soc. 1989, 111, 4613-4622.

19. Hunter, E. P. L.; Lias, S. G. Evaluated Gas-Phase Basicities and Proton Affinities of Molecules: An Update. J. Phys. Chem. Ref. Data 1998, 27, 413-656.

20. Meot-Ner, M.; Scheiner, S.; Yu, W. O. Hydrogen Bonds in Bioenergetics. 3. Proton Transport in Membranes, Modeled by Ketone/Water Clusters. J. Am. Chem. Soc. 1998, 120, 6980-6990.

21. Morrison, G. C.; Howard, C. J. Selective Detection of Gas-Phase Aldehydes and Ketones Using Protonated Hydrazine. Int. J. Mass Spectrom. 2001, 210/211, 503-509.

22. Morrison, G. C.; Custer, T. G.; Kato, S.; Bierbaum, V. M.; Howard, J. H. Gas-Phase Kinetics and Mechanism of the Reactions of Protonated Hydrazine with Carbonyl Compounds. J. Am. Chem. Soc. 2004, 126, 2744-2754.

23. Mason, E. A. Ion Mobility: Its Role in Plasma Chemistry. In Plasma Chromatography; Carr, T. W., Ed.; Plenum Press: New York, 1984; pp 43-93.

24. Bollan, H. R. The Detection of Hydrazine and Related Materials by Ion Mobility Spectrometry; Ph.D. Thesis, Sheffield-Hallum University, England, 1998, pp 90-95. 